\title{
A Novel Based Approach for iris code Recognization Using Gabor Filter
}

\author{
S.Tamilselvi, ${ }^{2}$ Dr. C. N. Marimuthu \\ ${ }^{I}$ Student of M.E, ${ }^{2}$ Dean/ECE Nandha Engineering College, Erode-52.
}

\begin{abstract}
Iris recognition is a part of biometric identification methodologies which also include facial, fingerprint, retinal and many other biological traits. They all over a new solution for person identification, authentication and security. Currently users have to carry security badges or know certain pin/pass codes in order to get into secure zones or to log into a computer. Problem with these methods is that users have to remember lots of different passwords and pin codes which therefore tend to be rather easy to guess and crack since users prefer passwords that are easy to remember. Cards can be lost and they can be used by anyone to gain access to a restricted area or to a restricted computer. Biometrics on the other hand provide a certain and easy way of authenticating persons, biometrics are also quite hard to forge and combined with some other method like password they form up a very strong authentication method.

Index terms: iris code, gabour filter, biometrics, feature extraction
\end{abstract}

\section{Introduction}

The iris is formed in early life in a process called morphogenesis. Iris recognition uses the random, colored patterns within the iris. These patterns are unique for each individual. The iris is the elastic, pigmented, connective tissue that controls the pupil. Once fully formed, the texture is stable throughout life. It is the only internal human organ visible from the outside and is protected by the cornea. The iris of the eye has a unique pattern, from eye to eye and person to person. An iris scan will analyze over 200 points of the iris, such as rings, furrows, freckles, and the corona and will compare it a previously recorded template. Glasses, contact lenses, and even eye surgery does not change the characteristics of the iris. To prevent an image / photo of the iris from being used instead of a real "live" eye, iris scanning systems will vary the light and check that the pupil dilates or contracts.

Principal component analysis is the simplest of the true eigenvector-based multivariate analyses. PCA is closely related to factor analysis [1]. CCA is the Once region boundaries have been detected, it is often useful to extract regions which are not separated by a boundary [2]. Any set of pixels which is not separated by a boundary is call connected. Each maximal region of connected pixels is called a connected component. The set of connected components partition an image into Segments. Image segmentation is a useful operation in many image processing applications [3]. A neural network consists of an interconnected group of artificial neurons, and it processes information using a connectionist approach tocomputation. In most cases a neural network is an adaptive systemthat changes its structure during a learning phase. Neural networks are used to model complex relationships between inputs and outputs or tofind patterns in data [4].

Principal component analysis will work with different parameters in the image in sequence manner [1].Connected component analysis will work with different parameters in the image in same time, but the output is not reliable for a large set of images [2].In neural network, for each and every time, the large set of feature for image database get loaded for training process, it will increase the time complexity of the whole system [3].

\section{Methodology}

In our proposed system we use the ICA (Independent component analysis) and Gabor filter to improve the sensitivity, specificity and reducing the time complexity in the existing system. The concept of Gabor filter will analyze the input image in several phases and pick a better one through 500 iterations. The number of iterations is our wish, according to the image size and image quality parameters. (500 iterations are recommended for natural images and 850 iterations are recommended for medical images).Independent component analysis is used to process the different parameters in the image in parallel manner 


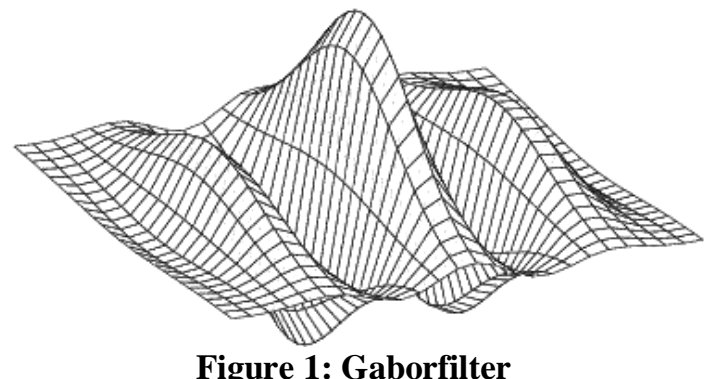

A Figure 1 shows the gabor filterThe real and imaginary components of a complex Gabor filter are phase sensitive,i.e., as a consequence their response to a sinusoid is another sinusoid. By getting the magnitude of the output (square root of the sum of squaredreal and imaginary outputs) we can get a response that phase insensitive and thusunmodulated positive response to a target sinusoid input.

\section{Block Diagram Of Decompression Algorithm}

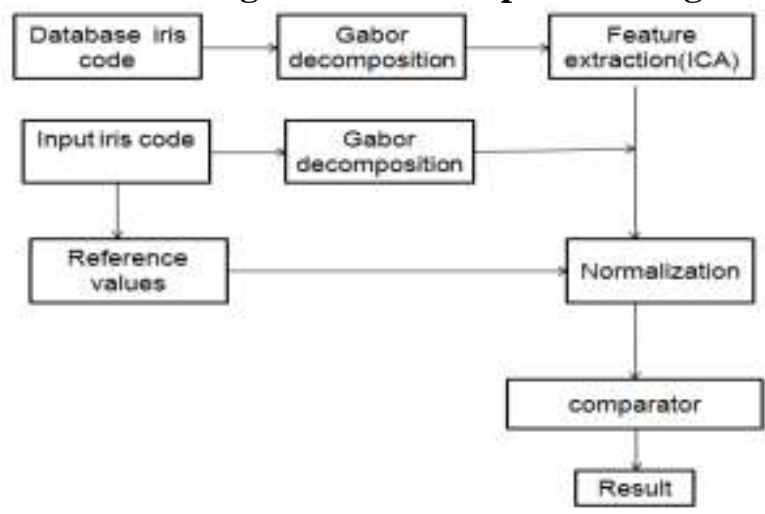

Figure 2: A schematic diagram of the decompressionscheme

A Figure 2 shows the schematic diagram of the decompression scheme. The black arrows indicate, respectively, the information flow of the decompression process and the information flow of the preparation of training images.

\section{MODULE 1:}

Module diagrams are used to show the allocation of classes and objects to modules in the physical design of a system, that is module diagrams indicate the partitioning of the system architecture.

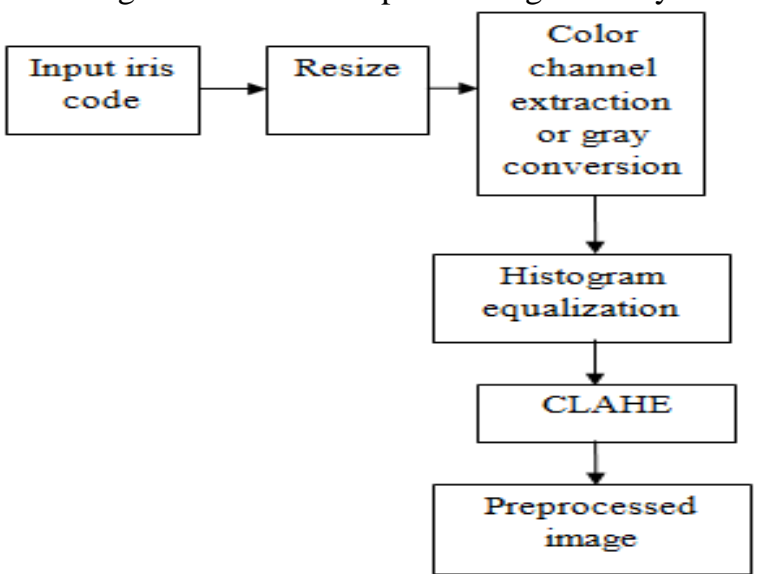

Figure 3: A schematic diagram of the preprocessing scheme

A Figure 3 showsthe schematic diagram of the preprocessing scheme. The black arrows indicate, respectively, the information flow of the preprocessing process and the information flow of the preparation of training images.

\section{A.INPUT IRIS CODE}

The Figure 4 shows the input image. The input iris image is a gray image. It is of default size $576 * 768$. For our convenience, the input iris image is resized to $512 * 512$. 


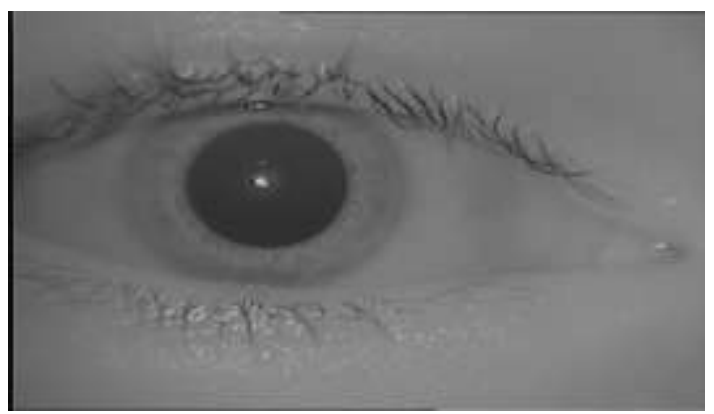

Figure 4: Input Image

\section{B. Gabor Decomposition}

Gabor filter will analyze the input image in several phases and pick a better one through 500 iterations. The number of iterations is our wish, according to the image size and image quality parameters.(500 iterations are recommended for natural images and 850 iterations are recommended for medical images).

\section{Data Base}

A database is a collection of information that is organized so that it can easily be accessed, managed, and updated. In one view, databases can be classified according to types of content: full-text, numeric, and images. The data are typically organized to model relevant aspects of reality in a way that supports processes requiring this information (for example, finding a hotel with vacancies).

\section{Feature Extraction}

In this feature extraction methods find out the Mean, Variance, Energy, skewness and Kurtosis. Mean

Average or mean value of array

\section{Variance}

Compute variance of input or sequence of inputs

\section{Skewness}

Skewness is a measure of the asymmetry of the data around the sample mean. If skewness is negative, the data are spread out more to the left of the mean than to the right. If skewness is positive, the data are spread out more to the right. The skewness of the normal distribution (or any perfectly symmetric distribution) is zero. The skewness of a distribution is defined as

$$
\mathrm{K}=\mathrm{E}(\mathrm{x}-\mu) 3 / \sigma 3 \ldots . . . .[1]
$$

Where $\mu$ is the mean of $\mathrm{x}, \sigma$ is the standard deviation of $\mathrm{x}$, and $\mathrm{E}(\mathrm{t})$ represents the expected value of the quantity t. skewness computes a sample version of this population value.

\section{Kurtosis}

Kurtosis is a measure of how outlier-prone a distribution is. The kurtosis of the normal distribution is 3. Distributions that are more outlier-prone than the normal distribution have kurtosis greater than 3; distributions that are less outlier-prone have kurtosis less than 3. The kurtosis of a distribution is defined as

$\mathrm{K}=\mathrm{E}(\mathrm{x}-\mu) 4 / \sigma 4$

Where $\mu$ is the mean of $\mathrm{x}, \sigma$ is the standard deviation of $\mathrm{x}$, and $\mathrm{E}(\mathrm{t})$ represents the expected value of the quantity t. kurtosis computes a sample version of this population value.

\section{Normalization}

Normalization is the process of taking data from a problem and reducing it to a set of relations while ensuring data integrity and eliminating data redundancy. Normalization is a property of the Sensitivity Analysis Options object. Use Normalization to specify the normalization for the computed sensitivities. Our input iris image is already in pure gray scale. Since no need of the conversion.

\section{F.CLAHE}

A variant of adaptive histogram equalization called Contrast Limited Adaptive Histogram Equalization (CLAHE) prevents this by limiting the amplification. 


\section{G.GRAY SCALE CONVERSION}

Our input iris image is already in pure gray scale. Since no need of the conversion.

\section{H.HISTOGRAM}

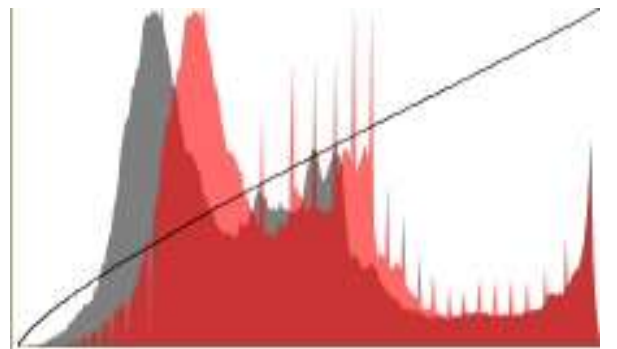

Figure 5: Histogramimage

An image histogram is a type of histogram that acts as a graphical representation of the tonal distribution in a digital image. It plots the number of pixels for each tonal value. By looking at the histogram for a specific image a viewer will be able to judge the entire tonal distribution at a glance.

Histogram modeling techniques (e.g. histogram equalization) provide a sophisticated method for modifying the dynamic range and contrast of an image. The Figure 5shows the histogram image.

Thehorizontal axis of the graph represents the tonal variations, while the vertical axis represents the number of pixels in that particular tone. The left side of the horizontal axis represents the black and dark areas, the middle represents medium grey and the right hand side represents light and pure white areas. The vertical axis represents the size of the area that is captured in each one of these zones. Thus, the histogram for a very bright image with few dark areas and/or shadows will have most of its data points on the right side and center of the graph. Conversely, the histogram for a very dark image will have the majority of its data points on the left side and center of the graph.

Image histograms can be useful tools for thresholding. Because the information contained in the graph is a representation of pixel distribution as a function of tonal variation, image histograms can be analyzed for peaks and/or valleys which can then be used to determine a threshold value. This threshold value can then be used for edge detection, image segmentation, and co-occurrence matrices.

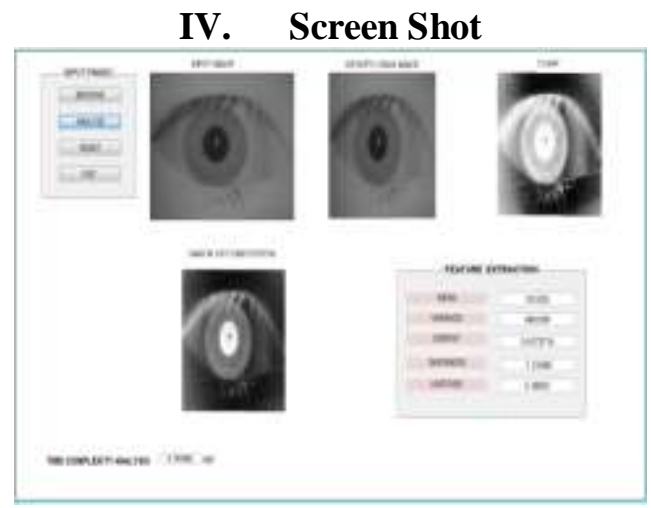

Figure 6: Left Eye Image

TheFigure6 shows that left eye resized image, CLAHE, Gabor filter and feature extraction values of Mean, Variance, Energy, Skewness and Kurtosis.

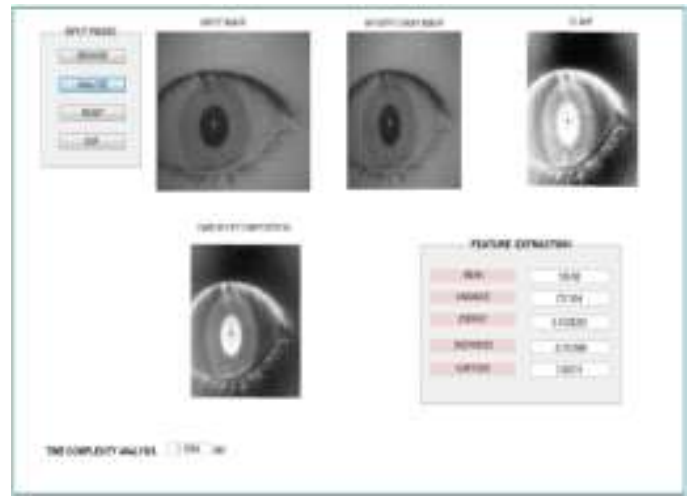

Figure 7:Right Eye Image 
TheFigure7 shows that Right eye resized image, CLAHE, Gabor filter and feature extraction values of Mean, Variance, Energy, Skewness and Kurtosis.

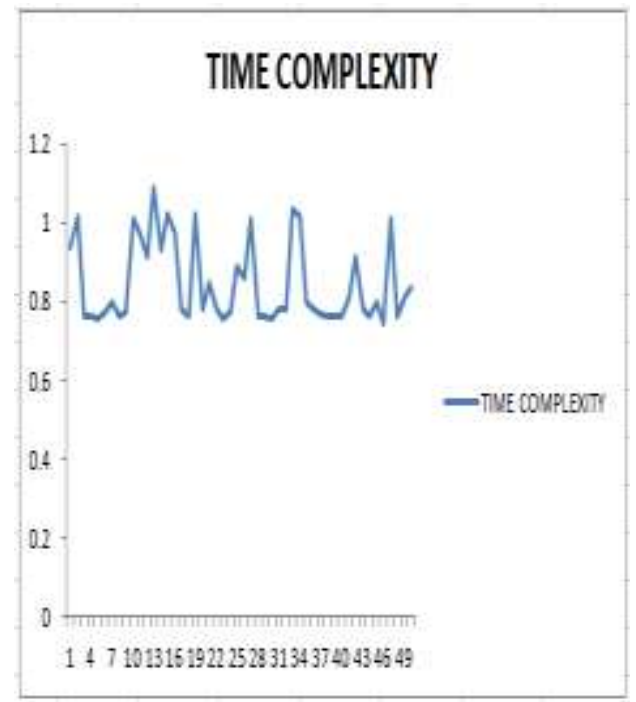

Figure 8: Left Eye Time Complexity

The Figure8 shows the Left eye Feature Extraction values. In this found reduce the $20 \%$ time complexity values.

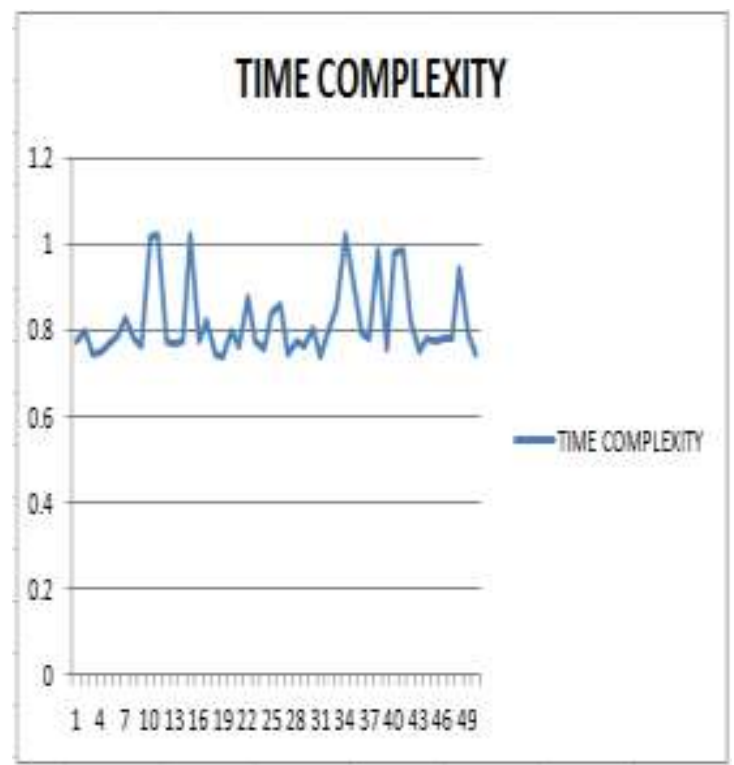

Figure 9: Left Eye Time Complexity

The Figure 9 shows the Right eye Feature Extraction values. In this found reduce $20 \%$ the time complexity values.

\section{Conclusion And Future Enhancement}

I proposed a method to reduce time complexity in iris Recognization. The concept of Gabor filter has analyzed the input image in several phases and picks a better one through 500 iterations. The number of iterations is designer wish, according to the image size and image quality parameters. (In base paper 500 iterations are recommended for natural images and 850 iterations are recommended for medical images).The proposed algorithm uses a bank of Gabor filters to capture both local and global iris characteristics to form a fixed length feature vector. In this method found the feature extraction values that are mean, variance, energy, skewness and kurtosis.

In future, the performance values, efficiency and sensitivity of the iris Recognization will be increased. The performance is compared to the biometrics techniques. 


\section{References}

[1] Adams Wai-Kin Kong, "Iris code Decompression Based on the Dependence between Its Bit Pairs," IEEE Transactions on Pattern Analysis and Machine Intelligence, Vol. 34, No. 3, March 2012.

[2] Daugman, J. "New Methods in Iris Recognition," IEEE Trans. System, Man, and Cybernetics Part B, vol. 37, no. 5, pp. 11671175,Oct. 2007.

[3] Shad .S and Ross .A, “Generating Synthetic Irises by Feature Agglomeration,” Proc. Int'l Conf. Image Processing, pp. 317-320,2006.

[4] Z. Sun, T. Tan, Y. Wang, and S.Z. Li, "Ordinal Palm print Representation for Personal Identification," Proc. IEEE CS Conf.Computer Vision and Pattern Recognition, vol. 1, pp. 279-284, 2005.

[5] Valentian .T. Ea, A., Rossant .F, Amiel, and A. Amara .F, “Algorithm Implementation for Iris Identification,” Proc. 48 ${ }^{\text {th }}$ Midwest Sump. Circuits and Systems, pp. 1207-1210, 2005.

[6] Kong. A, Zhang. D and Kamel. M, "Palm print Identification Using Feature-Level Fusion,” Pattern Recognition, vol. 39, pp. 478-487, 2006J. Daugman, "How Iris Recognition Works,” IEEE Trans. Circuits and Systems for Video Technology, vol. 14, no. 1, pp. 21 -30, Jan. 2004.

[7] Kong. A.W.K. and Zhang .D, “Competitive Coding Scheme for Palm print Verification,” Proc. Int'l Conf. Pattern Recognition, vol. 1, pp. 520-523, 2004.

[8] Tan. L. Ma, T, Wang. Y, and Zhang.D, "Efficient Iris Recognition by Characterizing Key Local Variations,” IEEE Trans. Image Processing, vol. 13, no. 6, pp. 739-750, June 2004

[9] Sun .Z, Tan .T, and Wang .T, "Iris Recognition Based on Non-Local Comparisons," Proc. Fifth Chinese Conf. Biometric Recognition, pp. 491-497, 2004.

[10] Krichen .E, Mellakh. M.A, Garcia-Salicetti. S, and Dorizzi .B, "Iris Identification Using Wavelet Packets,” Proc. Int'l Conf. Pattern Recognition, vol. 4, pp. 226-338, 2004.

[11] Zhang. P.F, Li. D.S, and Wang .Q, “A Novel Iris Recognition Method Based on Feature Fusion,” Proc. Third Int'1 Conf. Machine Learning and Cybernetics, pp. 26-29, 2004

[12] Daugman .J, “The Importance of Being Random: Statistical Principles of Iris Recognition,” Pattern Recognition, vol. 36, pp. 279-291, 2003. 